\title{
\$37. Inner products for protoscales
}

Given any protoscale $E$ and given any $B \in[1, O(\ell(E))]$

and $\mathrm{G} \subset \mathrm{Q}$ and $\mathrm{u} \in \mathrm{Q}$ we define

$$
\begin{aligned}
G(E(B) \geq u)=\{j \in G(l(E)): & \operatorname{inpo}(j, E(B, \tilde{B}, \hat{B}, \hat{C})) \geq u \\
& \text { for all }(\tilde{B}, \hat{B}, \hat{C}) \in \operatorname{supt}(E, B)\}
\end{aligned}
$$

and

$$
\begin{aligned}
G(E((B)) \geq u)=\{j \in G(l(E)): & \operatorname{inpo}(j, E((B, \tilde{B}, \hat{B}, \hat{C}))) \geq u \\
& \text { for all }(\tilde{B}, \hat{B}, \hat{C}) \in \operatorname{supt}((E, B))\}
\end{aligned}
$$

and for any $P \in\{>,=\}$ we define

$$
\begin{aligned}
& G(E(B) P u)=\{j \in G(E(B) \geq u): \\
& \text { inpo }(j, E(B, O(l(E)), O(l(E)), O)) P u\}
\end{aligned}
$$

and

$$
\begin{aligned}
& G(E((B)) P u)=\{j \in G(E((B)) \geq u): \\
&\quad \operatorname{inpo}(j, E((B, O(l(E)), O(\ell(E)), O))) P u\} .
\end{aligned}
$$

